NASA/TM-2000-210057

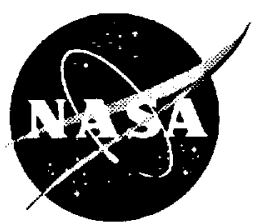

\title{
A Wideband Satcom Based Avionics Network With CDMA Uplink and TDM Downlink
}

D. Agrawal and B.S. Johnson

University of Illinois, Urbana, Illinois

U. Madhow

University of California, Santa Barbara, Santa Barbara, California

K. Ramchandran

University of California, Berkeley, Berkeley, California

K.S. Chun

Glenn Research Center, Cleveland, Ohio 
Since its founding, NASA has been dedicated to the advancement of aeronautics and space science. The NASA Scientific and Technical Information (STI) Program Office plays a key part in helping NASA maintain this important role.

The NASA STI Program Office is operated by Langley Research Center, the Lead Center for NASA's scientific and technical information. The NASA STI Program Office provides access to the NASA STI Database, the largest collection of aeronautical and space science STI in the world. The Program Office is also NASA's institutional mechanism for disseminating the results of its research and development activities. These results are published by NASA in the NASA STI Report Series, which includes the following report types:

- TECHNICAL PUBLICATION. Reports of completed research or a major significant phase of research that present the results of NASA programs and include extensive data or theoretical analysis. Includes compilations of significant scientific and technical data and information deemed to be of continuing reference value. NASA's counterpart of peerreviewed formal professional papers but has less stringent limitations on manuscript length and extent of graphic presentations.

- TECHNICAL MEMORANDUM. Scientific and technical findings that are preliminary or of specialized interest, e.g., quick release reports, working papers, and bibliographies that contain minimal annotation. Does not contain extensive analysis.

- CONTRACTOR REPORT. Scientific and technical findings by NASA-sponsored contractors and grantees.
- CONFERENCE PUBLICATION. Collected papers from scientific and technical conferences, symposia, seminars, or other meetings sponsored or cosponsored by NASA.

- SPECIAL PUBLICATION. Scientific, technical, or historical information from NASA programs, projects, and missions, often concerned with subjects having substantial public interest.

- TECHNICAL TRANSLATION. Englishlanguage translations of foreign scientific and technical material pertinent to NASA's mission.

Specialized services that complement the STI Program Office's diverse offerings include creating custom thesauri, building customized data bases, organizing and publishing research results ... even providing videos.

For more information about the NASA STI Program Office, see the following:

- Access the NASA STI Program Home Page at http://www.sti.nasa.gov

- E-mail your question via the Internet to help@sti.nasa.gov

- Fax your question to the NASA Access Help Desk at (301) 621-0134

- Telephone the NASA Access Help Desk at (301) 621-0390

- Write to: NASA Access Help Desk NASA Center for AeroSpace Information 7121 Standard Drive Hanover, MD 21076 
NASA/TM-2000-210057

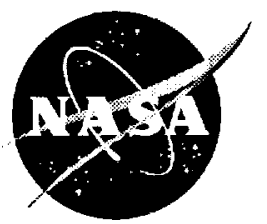

\section{A Wideband Satcom Based Avionics Network With CDMA Uplink and TDM Downlink}

D. Agrawal and B.S. Johnson

University of Illinois, Urbana, Illinois

U. Madhow

University of California, Santa Barbara, Santa Barbara, California

K. Ramchandran

University of California, Berkeley, Berkeley, California

K.S. Chun

Glenn Research Center, Cleveland, Ohio

Prepared for the

18th International Communications Satellite Systems Conference and Exhibit sponsored by the American Institute of Aeronautics and Astronautics

Oakland, California, April 10-14, 2000

National Aeronautics and

Space Administration

Glenn Research Center 
This report contains preliminary

findings, subject to revision as analysis proceeds.

Available from

NASA Center for Aerospace Information

7121 Standard Drive

Hanover, MD 21076

Price Code: A03
National Technical Information Service 5285 Port Royal Road Springfield, VA 22100

Price Code: A03 


\section{A Wideband Satcom Based Avionics Network With CDMA Uplink and TDM Downlink}

\author{
D. Agrawal and B.S. Johnson \\ University of Illinois \\ Urbana, Illinois
}

\author{
K. Ramchandran \\ University of California, Berkeley \\ Berkeley, California
}

\author{
U. Madhow \\ University of California, Santa Barbara \\ Santa Barbara, California
}

\section{Abstract}

The purpose of this paper is to describe some key technical ideas behind our vision of a future satcom based digital communication network for avionics applications The key features of our design are as follows:

(a) Packetized transmission to permit efficient use of system resources for multimedia traffic;

(b) A time division multiplexed (TDM) satellite downlink whose physical layer is designed to operate the satellite link at maximum power efficiency. We show how powerful turbo codes (invented originally for linear modulation) can be used with nonlinear constant envelope modulation, thus permitting the satellite amplifier to operate in a power efficient nonlinear regime.

(b) A code division multiple access (CDMA) satellite uplink, which permits efficient access to the satellite from multiple asynchronous users. Closed loop power control is difficult for bursty packetized traffic, especially given the large round trip delay to the satellite. We show how adaptive interference suppression techniques can be used to deal with the ensuing near-far problem.

(c) Joint source-channel coding techniques are required both at the physical and the data transport layer to optimize the end-to-end performance. We describe a novel approach to multiple description image encoding at the data transport layer in this paper.

\section{Introduction}

An upgrade of the telecommunications infrastructure for avionics applications will require the use of advanced digital communication methods which support a diverse mix of traffic requirements, including voice, data, image, and video transmission. Such a system would involve not only communication between aircraft and ground stations, but also between aircraft and aircraft. Satellite communication forms an attractive option for providing global connectivity in this scenario. This paper reports on a research effort aimed at designing the physical layer for a satcom based digital avionics network. We propose to utilize the Ku and Ka bands. Since the link to and from the satellite is expected to form the bottleneck, we focus on the design of that link in this paper. In order to minimize the interference caused to other satcom systems in the same band, spread spectrum transmission will be employed, so that the transmitted power is spread over the entire available bandwidth. Furthermore, in order to facilitate support of multimedia traffic, packet-based transmission will be used. Some of the salient features of the design, and of the results presented in the paper, are summarized below.

Downlink: The one-to-many "downlink" from the satellite is severely powerlimited, with the result that the power amplifiers in the satellite typically operate in a nonlinear saturation mode. Hence, constant envelope modulation will be employed on the downlink. Continuous phase modulation (CPM), which is a nonlinear modulation strategy, is a bandwidth efficient strategy in this context. However, it has not become as popular as linear modulation strategies, because the state of the art of coding techniques for CPM is not advanced. In Section 2 of this paper, we show how powerful turbo-like codes, developed originally for binary linear modulation, can be leveraged into CPM systems.

Uplink: Direct sequence (DS) code division multiple access (CDMA) with linear data modulation 
will be employed for the many-to-one "uplink" to the satellite. The data for each user modulates a user-specific wideband spreading waveform. We employ short spreading waveforms, in which the period of the spreading waveform equals the symbol interval. This is in contrast to the CDMAbased US digital cellular standard, IS-95, which employs "long" spreading waveforms whose periods are much longer than the symbol interval. Short spreading sequences are chosen because they are compatible with adaptive interference suppression schemes $[1,2,3,4]$ that provide large gains in performance and capacity compared to conventional systems based on long spreading waveforms. In Section 3 of this paper, we provide a tutorial on recent developments in adaptive interference suppression, and give new results on joint Doppler estimation and interference suppresion.

Joint Source-Channel Coding: Separate source coding and channel coding is optimal in an informationtheoretic sense (i.c., with no delay or complexity constraints) for singleuser transmission over a timeinvariant channel. However, joint source-channel coding is important for optimizing end-to-end Quality of Service in many practical settings, including real-time traffic, multiple users, and time-varying channels. This is especially crucial for wireless image and video transmission. In Section 4 of this paper, we describe a source-channel coding strategy for end-to-end quality of service on a packetized network. Ongoing work on source-channel coding for a single satellite link is not described.

After describing the main technical results in Sections 2 through 4 , we give our conclusions in Section 5 .

\section{Forward Link: Turbo Coded CPM}

Continuous phase modulation (CPM) signaling provides bandwidth-efficient constant envelope modulation schemes that suitable for downlinks from satellites. The excess phase of such signals varies continuously and is given by the convolution of an $M$-ary data sequence $\left\{\alpha_{i}\right\}$ with a phase response function $q(t)$. Thus, a CPM signal can be described by

$$
\begin{gathered}
s(t)=\sqrt{\frac{2 E}{T}} \cos \left(2 \pi f_{0} t+2 \pi h \sum_{i=0}^{n} \alpha_{i} q(t-i T)\right) \\
n T \leqslant t<(n+1) T
\end{gathered}
$$

where $h=K / P$ is the modulation index and $T$ is the symbol duration. Usually, $M=2^{k}$ for an integer $k$, and $\alpha_{i}$ 's take values in the set $\{ \pm(M-$ $1), \pm(M-3), \ldots, \pm 1\}$. Denote the excess phase of the signal given above by $\phi(t)$, that is,

$$
\phi(t)=2 \pi h \sum_{i=0}^{n} \alpha_{i} q(t-i T) \quad n T \leqslant t<(n+1) T
$$

At present, some of the best coding schemes for continuous phase modulation use convolutional codes based on $\mathbb{Z}_{P}$, the ring of integers modulo $P$. For good performance these convolutional codes have to be judiciously chosen for each specific CPM signaling according to the parameters $h$ and $M$, and the phase response function $q(t)[5]$. Furthermore, the choice of codes is greatly influenced by the channel and receiver characteristics.

Recently discovered graph-based codes (most notably turbo codes) and associated iterative decoding algorithms have demonstrated coding gains that far exceed the coding gains provided by convolutional codes. In fact, for a wide class of binary-input channels and receivers, these codes can achieve performance close to the capacity [6]. However, graphbased codes are usually compatible with binaryinput channels, and they cannot directly be applied to an $M$-ary input continuous phase modulated (CPM) channel. Arguably, it is possible to extend binary graph-based codes to $M$-ary alphabets in order to use them with CP.M. This approach, however, will not be able to capitalize on the vast research being done on binary graph-based codes. More importantly, such an approach may be unnecessary as long as an $M=2^{k}$-ary input CPM channel can be divided into parallel binary-input channels.

In this paper, we use bit interleaved coded modulation (BICM) to convert an $M$-ary CPM channel into several binary-input channels [7], and thus enable graph-based coded modulation on CPM channels. In Section 2.1, we briefly describe a BICM system model that uses CPM channels. Next, in Section 2.2, we describe the results of our extensive simulations. 


\subsection{System Model}

Figure 1 shows the block diagram of a communication system which uses bit interleaved coded modulation with a CPM channel. In this setup, the binary codeword at the output of the channel encoder is fed to a bit interleaver $\pi$. The output of bit interleaver $\pi$ is then passed to a (one-to-one) labeling mapper $\mu$ which maps a group of $N k$ bits to a sequence of $N$ symbol in an $M=2^{k}$-ary input alphabet $\mathcal{X}$. Denote the resulting sequence of length $N$ by $\mathbf{x}$. The sequence $\mathbf{x}$ is transmitted using continuous phase modulation. Note that due to the intersymbol interference introduced by the phase response function $q(t)$, a continuous phase modulator is not memoryless, rather, it can be modeled as a finite state machine. We assume that in the beginning of transmission (of each sequence) the modulator state is zero, and at the end of each sequence, some extra symbols are sent to reset the modulator state back to zero.

At the receiver, the received signal $\mathbf{y}$ is passed to a demodulator which computes the bitwise soft information $p\left(\mathbf{y} \mid l^{i}(\mathbf{x})=b\right)$. Here $l^{i}(\mathbf{x})$ denotes the $i$-th bit of $\mu^{-1}(\mathbf{x})$ and $b$ belongs to the set $\{0,1\}$. Finally, the bitwise soft information is deinterleaved and is passed to a turbo decoder. Using the analysis of BICM by Caire, Taricco, and Biglieri [7], it is clear that, under the assumption of ideal interleaving, the above arrangement converts an $M$-ary input $\mathrm{CPM}$ channel into $\mathrm{Nk}$ parallel, independent, memoryless, binary-input channels. These channels have roughly the same SNR and noise characteristics.

Note that, apart from the CPM demodulator, this setup uses standard off-the-shelf components, and thus reduces the implementation complexity. In the following subsection, we will briefly describe an easily implementable CPM demodulator which can generate bitwise soft information. This demodulator is a straight-forward result of combining Rimoldi's time-invariant trellis of CPM signals with the Bahl-Coke-Jelinek-Raviv (BCJR) algorithm. For more details on these, the reader is referred to [8], [9].

\subsubsection{CPM demodulator with bitwise soft decisions}

It is well known that continuous phase modulated signals given by (1) can be represented by a timevariant trellis that describes the evolution of the

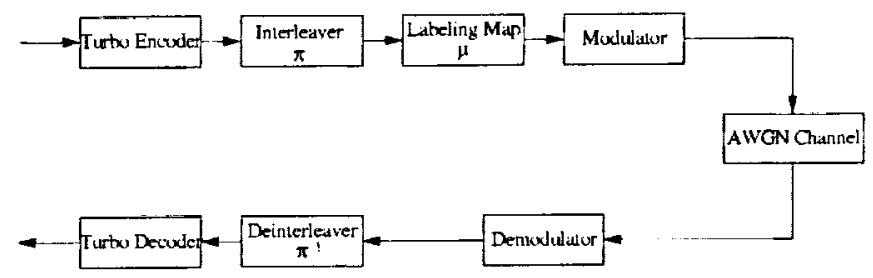

Figure 1: A turbo-coded and continuous phase modulated communication system

excess phase $\phi(t)$. In principle, we can obtain the bitwise soft information by applying the BCJR algorithm on this trellis with a complexity that grows only linearly in the number of bits [9]. However, the time-variant nature of the trellis makes the termination of the trellis in the zero state at the end of $N k$ bits difficult and the large number of states $2 P M^{L-1}$ in such a trellis causes extra computation complexity. Fortunately, an alternative representation of continuous phase modulation, proposed by Rimoldi [8], results in a time-invariant trellis with lesser number of states.

In Rimoldi's formulation, instead of using (1) to construct phase trellis, an alternative representation of the CPM signal is used [8]. In this representation, the center frequency is chosen to be $f_{1}=f_{0}-h(M-1) /(2 T)$ so that the excess phase of a CPM signal becomes, for $t=n T+\tau(0 \leqslant \tau<T)$, $\psi(t=n T+\tau)=2 \pi h \sum_{i=0}^{n} \alpha_{i} q(t-i T)+\frac{h(M-1)}{2 T}$ $=2 \pi h \sum_{i=0}^{n-L} U_{i}+4 \pi h \sum_{i=0}^{L-1} U_{n-i} f(\tau+i T)+W(\tau)$

where

$$
\begin{aligned}
& W(\tau)=\frac{\pi h(M-1) \tau}{T}-2 \pi h(M-1) \sum_{i=0}^{L-1} f(\tau+i T) \\
& \quad+(L-1)(M-1) \pi h
\end{aligned}
$$

represents data independent terms, and $U_{i}=\left(\alpha_{i}+\right.$ $(M-1)) / 2$ take integer values between 0 and $M-1$. It was shown in [8] that the excess phase given by (2) can be represented on a time-invariant trellis with $P M^{L-1}$ states. Since this trellis is timeinvariant and includes 0 in its symbol alphabet, it can be easily terminated in the zero state.

At the receiver, the received signal is lowpass filtered and is sampled every $T / \xi$ second, where $\xi$ is the oversampling factor. These samples are passed to a CPM demodulator which computes soft information of the transmitted bits by using the standard BCJR algorithm on the time-invariant trellis 
described above. For more details on branch metric calculations and the effect of parameter $\xi$, we refer the reader to [10].

\subsection{Simulation Issues and Results}

In order to demonstrate the performance of CPM modulated channels, we used classical turbo codes with identical constituent convolutional codes generated by the polynomials $\left\{D^{4}+D^{3}+D^{2}+D^{1}+\right.$ $\left.1, D^{4}+1\right\}$. The parity bits were punctured alternatively to generate a rate $1 / 2$ turbo code. The interleaver length was $2^{15}=32768$. Turbo code with the aforementioned parameters has been evaluated on a variety of channels and it serves at a good performance benchmark.

A CPM signaling scheme can be specified by three parameters: number of data symbols $M$, the frequency response pulse $g(t)=d q(t) / d t$ and the modulation index $h$. We tested the performance of CPM channels for $M=2,4$ and 8 . In this paper, we provide results only for $M=4$ and 8 . It is well known that for $M=2, C P M$ is an inferior modulation scheme when compared to the CPM for $M=4$ and 8 [10, page 182]. Our simulation results affirm this fact for turbo-coded CPM channels also.

For full response signaling, we simulated continuous phase frequency shift keying (CPFSK) which is a special case of CPM modulation with rectangular frequency pulses. For partial response signaling, we used raised cosine frequency pulses of duration $3 T$ (also referred to as 3RC CPM signaling), where $T$ is the symbol period [10, page 52$]$.

The choice of modulation index $h=K / P$ is more involved. For a given $M$ and $g(t)$, increasing $h$ results in larger occupied bandwidth. However, increasing $h$ up to a certain limit, usually also results in larger minimum distance between CPM signals. Since the minimum distance dictates uncoded bit-error rate at high SNR's, by increasing $h$, one can trade-off bandwidth with transmitted energy (at least for high SNR's). After a certain limit, there is no benefit in increasing $h$ since that does not yield larger minimum distance [10, Chapter 3].

However, the trend of increasing minimum distance with $h$ does not hold for certain values of $h$, called weak modulation indices. Weak modulation indices generate CPM signals whose excess phases differ by a multiple of $2 \pi$ and therefore are identical to each other. This results in low minimum distance. Such weak modulation indices are usually

\begin{tabular}{|l|c|c|c|}
\hline \multicolumn{1}{|c|}{$M$} & 2 & 4 & 8 \\
\hline CPFSK & $\frac{7}{10}$ & $\frac{9}{10}$ & $\frac{9}{10}$ \\
\hline $3 \mathrm{RC}$ & $\frac{4}{5}$ & 1 & 1 \\
\hline
\end{tabular}

Table 1: Optimum values of $h$ for different CPM parameters

avoided in practice since the corresponding CPM schemes have low asymptotic gains.

For turbo-coded CPM signaling, the trade-offs described above are invalid because turbo-codes work at low SNR's, where apart from the minimum distance between CPM signals, second order effects are also as important. As a rule of thumb, for turbo codes to work well, the uncoded bit error rate (BER) should be between 10-15\%. Thus, we are interested in the SNR required by CPM schemes (with different $h$ ) to achieve a bit error rate of $13 \%$. Moreover, we are also interested in the values of $h$ that have low $P$, since the complexity of demodulator is directly proportional to $P$. In order to choose $h$, therefore, we simulated uncoded CPM for different values of $h$, and chose an $h$ that achieved a good trade-off between the value of $P$ and the SNR required to achieve a $13 \%$ bit error rate. For $M=2,4$ and 8 , and for CPFSK and 3RC, these values are given in Table 2.2. Note that for 3RC scheme, we have chosen weak modulation indices for $M=4$ and 8 .

Figures 2-5 show the simulated performance of turbo-coded CPM signaling. These figures also show the corresponding performances of turbo-coded $M$ ary PSK modulation. Note that, while PSK modulation produces a constant envelope signal in discrete time, the analog signal corresponding to PSK modulation, when used with a bandwidth efficient pulse such as a raised cosine, is not constant envelope. Thus, the satellite amplifier would need to back off from the saturation regime to support PSK, leading to a power penalty that is not accounted for here. Figure 2 shows that for $M=4, \mathrm{CPM}$ takes about $1.6 \mathrm{~dB}$ more SNR to achieve a bit-error rate of $10^{-5}$. However, this would be more than compensated by the ability to operate the satellite amplifier 


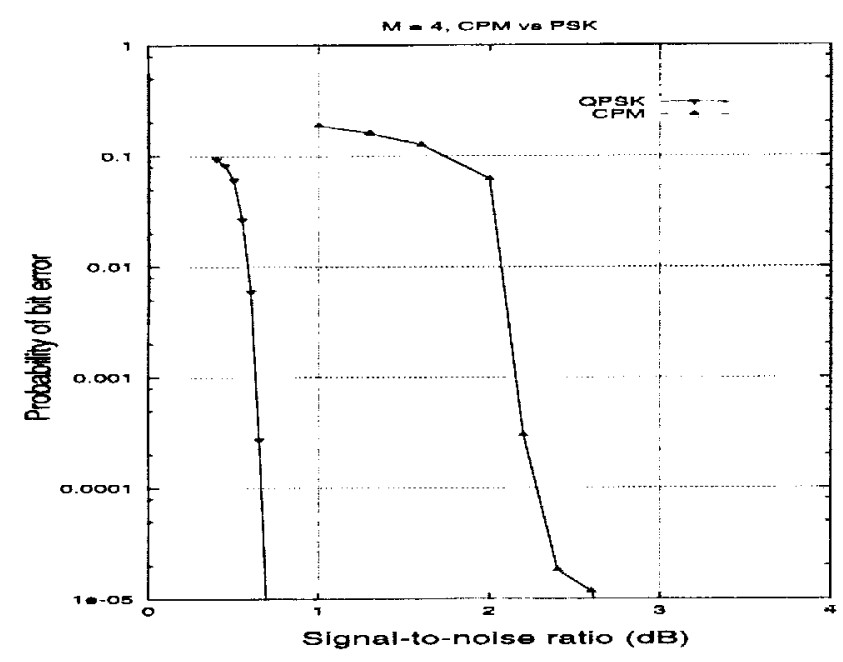

Figure 2: Turbo-coded CPFSK with $\mathrm{M}=4$ versus turbo-coded QPSK

in the nonlinear regime for CPM. Furthermore, note that power spectrum of CPM scheme is much more compact than the QPSK scheme. The power spectrum of the CPM signaling can be made even more compact without increasing transmitted power by using the 3RC CPM signaling (see Figure 3).

Even without accounting for the satellite amplifier operating regime, the penalty in transmitted power with respect to PSK modulation virtually disappears for $M=8$. Figures 4 and 5 show that in order to achieve a bit-error rate of $10^{-5}$, both CPM and PSK signaling use about the same transmitted power. However, the use of CPM signaling results in a much better power spectrum characteristics. We note that for $M=8$, the number of states in the CPM trellis is 10 for CPFSK and is 64 for $3 \mathrm{RC}$. On the other hand, the spectral characteristics of 3RC CPM signaling are much better than those of CPFSK signaling [10, page 147] Thus 3RC CPM signaling provides better spectrum at the cost of more complexity in the receiver.

\section{Reverse Link: Asynchronous CDMA with Interference Suppression}

In a DS-CDMA system, the information-bearing signal for each user is spread over a wider bandwidth by multiplying by a spreading waveform unique to that user. Multiple access capability is obtained

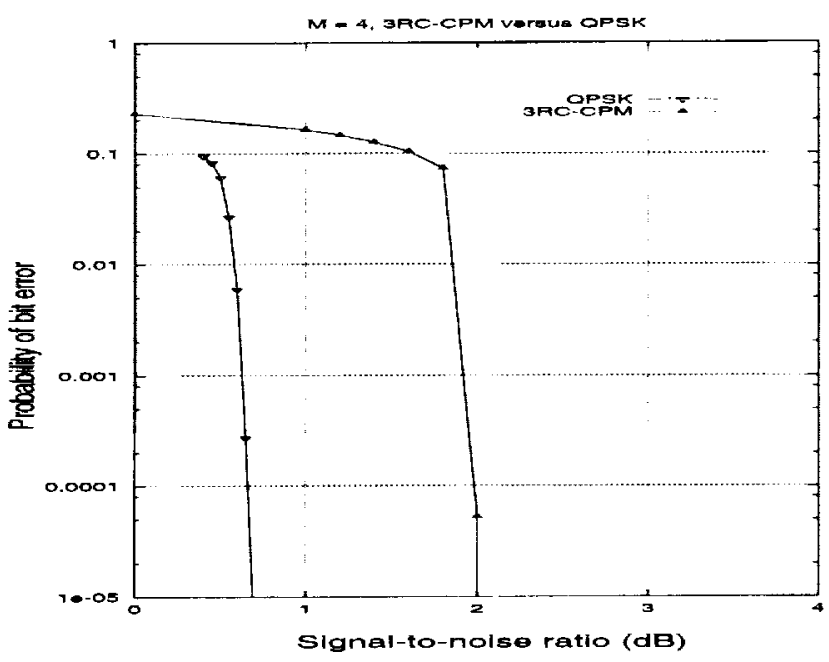

Figure 3: Turbo-coded 3RC CPM with $\mathrm{M}=4$ versus turbo-coded QPSK

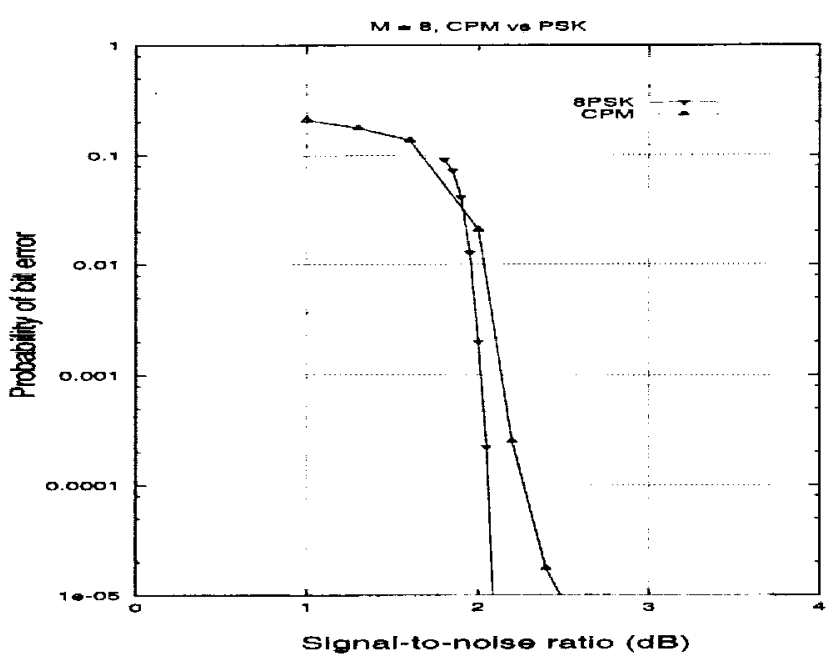

Figure 4: Turbo-coded CPFSK with $\mathrm{M}=8$ versus turbo-coded 8-PSK 


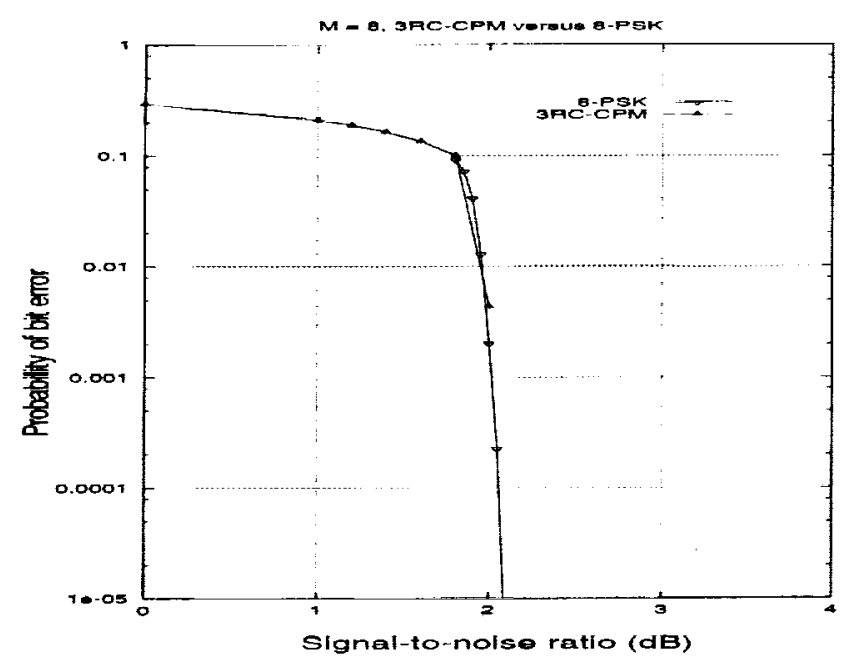

Figure 5: Turbo-coded 3RC CPM with $\mathrm{M}=8$ versus turbo-coded 8-PSK

by designing the spreading waveforms to have small crosscorrelations. Conventional DS-CDMA systems (as in IS-95) use spreading waveforms whose period is much larger than the symbol duration, and rely on interference averaging to provide robust performance. However, if the spreading waveforms have period equal to the symbol interval (these are termed short spreading codes), the multiple-access interference (MAI) becomes cyclostationary, and it is possible in theory to obtain much better performance than for conventional systems by learning the structure of interference, i.e., by using adaptive interference suppression. However, a key bottleneck to realizing the potential gains from adaptive interference suppression is that conventional adaptive algorithms cannot cope with the fast frequencyselective fading typical of wireless channels. Howcver, we have recently obtained algorithms, based on an invention termed the differential Minimum Mean Squared Error (DMMSE) criterion [4], that are robust over wireless channels. With this recent invention, a practical system architecture based on adaptive interference suppression is within reach. This technology also combines naturally with antenna arrays (using either beamforming or diversity techniques) to further enhance capacity and performance. Finally, the use of linear interference suppression reduces the multiuser CDMA channel approximately to a singleuser AWGN channel with fading, which means that work on the hot topic of source and channel coding for singleuser wireless channels can be leveraged into our application.
The new research reported in this paper consists of extending the DMMSE criterion to provide carrier frequency acquisition as well as interference suppression using digital signal processing, after bringing the received signal down to baseband using a fixed analog carrier generator. The satellite uplink is well modeled as a multiuser Additive White Gaussian Noise (AWGN) channel, with each user undergoing a (possibly different) Doppler shift. However, we present algorithms that can cope not only with a Doppler shift, but also with variations in amplitude and phase due to fading. This is so that the set of algorithms developed are applicable to terrestrial wireless settings as well, as might be the case in communication between aircraft and control towers.

Frequency acquisition consists of estimating and correcting for the Doppler offset suffered by the user of interest. We employ the DMMSE algorithm to provide interference suppression together with frequency acquisition. If the channel does not exhibit significant fading, then DMMSE adaptation can be replaced by standard MMSE adaptation.

In this section, we describe algorithms based on the DMMSE criterion for a general system model, of which asynchronous CDMA with short spreading sequences is a special case. This is followed by a description of the current work on extending these ideas to provide frequency acquisition as well as interference suppression.

\subsection{System Model for Adaptive In- terference Suppression}

It is useful to consider a generic model for interference suppression (which applies to other contexts such as equalization or beamforming as well) described in complex baseband, discrete time, notation. We will later describe how asynchronous CDMA with short spreading sequences is a special case of this model.

It is assumed that the received signal has already been passed through an appropriate filter and sampler. A linear receiver $c$ operates on a sequence of received vectors $\left\{\mathbf{r}_{n}\right\}$. Each received vector $\mathbf{r}_{n}$ is an $L$-dimensional complex vector which is a sum of a number of signal vectors, plus noise, where each signal vector corresponds to ISI or MAI. The output at time $n$ of the linear receiver $\mathbf{c}$ to be designed 
is given by $\left\langle\mathbf{c}, \mathbf{r}_{n}>=\sum_{i=1}^{L} c^{*}[i] r_{n}[i]\right.$, where

$$
\mathbf{r}_{n}=B_{1}[n] \mathbf{u}_{1}+\sum_{k=2}^{K} B_{k}[n] \mathbf{u}_{k}+\mathbf{w}_{n} .
$$

Here $\mathbf{u}_{1}$ is the desired signal vector, and $\mathbf{u}_{1}, \ldots, \mathbf{u}_{J}$ are interference vectors. These are assumed to remain roughly the same (or to vary slowly) with $n$. The terms $\left\{B_{k}[n], k=1, . ., K\right\}$ can vary arbitrarily with $n$, and their interpretation depends on the application (see Sections 4.1.3 and 4.1.4 for two specific applications). The vector $\mathbf{w}_{n}$ is discrete-time noise.

The standard linear MMSE receiver minimizes the Mean Squared Error (MSE) between the receiver output and the desired sequence $B_{1}[n]$, given by $E\left[\left(<\mathbf{c}, \mathbf{r}_{n}>-B_{1}[n]\right)^{2}\right]$. Such a receiver can be implemented adaptively using a number of well known algorithms [11]. The adaptive algorithms are initialized by a known training sequence $\{t[n]\}$ . Assuming that the desired sequence $\left\{B_{1}[n]\right\}$ satisfies $B_{1}[n]=g[n] t[n]$, where $g[n]$ is an unknown gain, and that $g[n]$ varies slowly with $n$, standard adaptive algorithms based on the cost function $E[(<$ $\left.\left.\mathbf{c}, \mathbf{r}_{n}>-t[n]\right)^{2}\right]$ can automatically compensate for the unknown gain $g[n]$. However, this procedure becomes difficult or impossible to implement when the variations in the unknown gain $g[n]$ are too rapid for the adaptive algorithm to compensate for them.

\subsection{Differential MMSE Detection}

In the applications of interest, while the desired sequence $\left\{B_{1}[n]\right\}$ includes an unknown gain $g[n]$, the gain $g[n]$ is roughly constant over two observation intervals. Thus, we assume that $A_{1}[n]=\frac{B_{1}[n]}{B_{1}[n-1]}$ is either known (because of training) or can be estimated. The key aspect of our scheme is that we choose the correlator $\mathbf{c}$ to satisfy the following differential MMSE criterion:

Minimize

$$
E\left[\left(<\mathbf{c}, \mathbf{r}_{n}>-A_{1}[n]<\mathbf{c}, \mathbf{r}_{n-1}>\right)^{2}\right]
$$

subject to

$$
E\left[\left|<\mathbf{c}, \mathbf{r}_{n}>\right|^{2}\right]=\mathrm{c}^{H} \mathbf{R} \mathbf{c}=1
$$

where $\mathbf{R}=E\left[\mathbf{r}_{n} \mathbf{r}_{n}^{H}\right]$, and where $\mathbf{x}^{H}$ denotes the conjugate transposed of $\mathbf{x}$.

It can be shown that, under standard assumptions, the solution to the above problem is the same as that for the conventional MMSE problem. However, our reformulation enables us to avoid tracking the unknown gain in the $B_{1}[n]$ by using $B_{1}[n-1]$ as a reference. Standard optimization techniques can be used to obtain a number of adaptive algorithms, a few of which are stated below.

\subsection{DMMSE-Based Algorithms}

The solution to the optimization problem (4)-(5) is the generalized eigenvector with the largest eigenvalue for the following generalized eigenvalue problem:

$$
\mathbf{B c}=\nu \mathbf{R c}
$$

where $\mathbf{B}=E\left[A_{1}^{*}[n] \mathbf{r}_{n} \mathbf{r}_{n-1}^{H}+A_{1}[n] \mathbf{r}_{n-1} \mathbf{r}_{n}^{H}\right]$.

\section{Block Algorithms}

Replacement of statistical expectations by empirical averages over $M$ observation intervals leads to block algorithms. This is analogous to the block LS algorithm for standard MMSE adaptation.

1. Compute $\hat{\mathbf{R}}=\sum_{n=1}^{M} \mathbf{r}_{n} \mathbf{r}_{n k}^{H}$ and $\hat{\mathbf{B}}=\sum_{n=1}^{M} A_{1}^{*}[n]$ $\mathbf{r}_{n} \mathbf{r}_{n-1}^{H}+A_{1}[n] \mathbf{r}_{n-1} \mathbf{r}_{n}^{H}$.

2. Use any standard algorithm to find the largest generalized eigenvector $\mathbf{c}$ for the pair $(\hat{\mathbf{B}}, \hat{\mathbf{R}})$. One possible algorithm is the power algorithm [12] applied to $\hat{\mathbf{R}}^{-1} \hat{\mathbf{B}}$.

\section{Recursive Algorithms}

Instead of averaging over a block of data, the averages can be performed recursively, using an exponential forget factor $0<\lambda \leqslant 1$. This is analogous to the RLS algorithm for standard MMSE adaptation.

1. Update $\hat{\mathbf{R}}_{n}=\lambda \hat{\mathbf{R}}_{n-1}+(\mathbf{1}-\lambda) \mathbf{r}_{n} \mathbf{r}_{n}^{H}$ and $\hat{\mathbf{B}}_{n}=$ $\lambda \hat{\mathbf{B}}_{n-1}+(1-\lambda)\left[A_{1}^{*}[n] \mathbf{r}_{n} \mathbf{r}_{n-1}^{H}+A_{1}[n] \mathbf{r}_{n-1} \mathbf{r}_{n}^{H}\right.$.

2. Update the largest generalized eigenvector $\mathbf{c}_{n}$ for the pair $\left(\hat{\mathbf{B}}_{n}, \hat{\mathbf{R}}_{n}\right)$. One possibility is to apply one or more steps of the power algorithm [12] on the matrix $\hat{\mathbf{R}}_{n}^{-1} \hat{\mathbf{B}}_{n}$, where $\hat{\mathbf{R}}_{n}^{-1}$ is updated using a standard RLS mechanism [11].

Alternatives to the preceding are obtained by updating $\mathbf{c}$ using a stochastic gradient for the cost function (4), together with a normalization that projects $\mathbf{c}$ approximately onto the constraint surface given by (5). This is analogous to the LMS or normalized LMS (NLMS) algorithm.

$$
\mathbf{c}_{n}=\frac{\mathbf{c}_{n-1}+\frac{\mu}{M_{n}} \mathbf{r}_{n}<\mathbf{c}_{n-1}, \mathbf{r}_{n-1} A_{1}[n]-\mathbf{r}_{n}>}{\sqrt{\mathbf{c}_{n-1}^{H} \hat{\mathbf{R}} \mathbf{c}_{n-1}}}
$$


where

$$
M_{n}=\lambda M_{n-1}+(1-\lambda) \mathbf{r}_{n}^{H} \mathbf{r}_{n}
$$

estimates the power of the received vector, and and where $\hat{\mathbf{R}}$ is an estimate of the correlation matrix $\mathbf{R}$.

\subsection{Application to CDMA with short sequences}

For a CDMA system in which user $k$ uses spreading waveform $s_{k}(t)$ to modulate all its transmitted symbols, the transmitted baseband signal for user $k$ is given by $\sum_{n} b_{k, n} s_{k}(t-n T)$, where $b_{k, n}$ is the $n$th symbol of the $k$ th user. This model applies both to narrowband systems with ISI and co-channel interference, and to wideband CDMA systems with short spreading sequences. For simplicity of exposition, we restrict attention to frequency nonselective fading in this report. Assuming that the fading is slow relative to the symbol interval, the received signal for the $k$ th user may be modeled as $\sum_{n} b_{k, n} F_{k}[n]\left(s_{k} * g_{k}\right)(t-n T)$, where $\left\{F_{k}[n]\right\}$ is a sequence of complex numbers modeling amplitude and phase fluctuations due to fading, and $g_{k}(t)$ is a normalized channel impulse response which is well modeled as time invariant (on the time scale of the receiver adaptation).

After filtering (typically using a chip matched filter) and sampling (typically at the chip rate or a multiple thereof), the received signal is of the form given by (3), where the time index $n$ corresponds to the symbol time, the desired sequence $B_{1}[n]=F_{1}[n] b_{1, n}$, and with the desired vector $\mathbf{u}_{1}$ denoting the sampled and windowed (by the filter length) version of the cascade of the spreading waveform and the channel seen by the desired user. For $k=2, \ldots, K$, the sequences $\left\{B_{k}[n]\right\}$ and the vectors $\mathbf{u}_{k}$ represent contributions due to ISI and MAI. See [3] for a tutorial description of the modeling involved, and see [1], [13], [2] for other papers using this equivalent synchronous model.

Assuming that the fading is roughly constant over two successive symbols (i.e., $F_{1}[n] \approx F_{1}[n-1]$ ), we have that $B_{1}[n] \approx A_{1}[n] B_{1}[n-1]$, where $A_{1}[n]=$ $b_{1, n} / b_{1, n-1}$ is the sequence of symbols used to train the DMMSE detector. the desired user. Algorithms based on the differential MMSE criterion can now be directly applied, using an initial training sequence for $\left\{b_{1, n}\right\}$, followed by decision-directed adaptation. The DMMSE detector leaves a phase ambiguity at the output, that can be resolved either by differential demodulation or by the use of pilot symbols. In standard differential demodulation, decisions on $A_{1}[n]=a_{1, n}$ would typically be based on the metric $\left\langle\mathbf{c}, \mathbf{r}_{n}><\mathbf{c}, \mathbf{r}_{n-1}\right\rangle^{*}$. However, it is also possible to get better performance for channels with coherence times of several symbols by using block noncoherent demodulation. A theory of signal design for such channels has recently been developed by Prof. Madhow [14], and will be leveraged into the current project as appropriate.

The receiver structure is depicted in Figure 6.

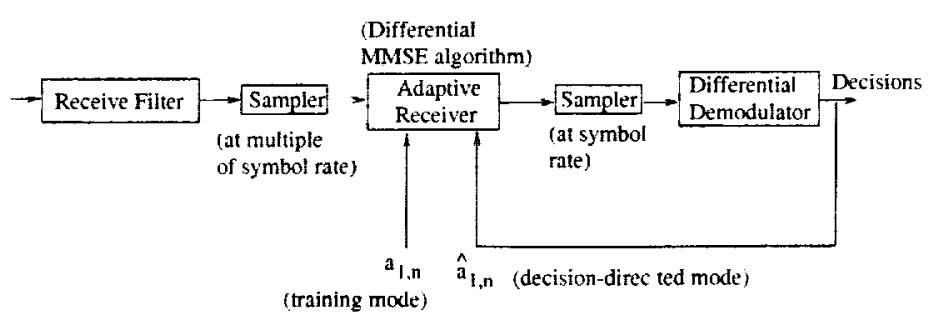

Figure 6: Receiver structure for interference suppression for CDMA with short spreading sequences. The receiver operates on samples at a multiple of the symbol rate.

\subsection{Frequency acquisition}

A frequency offset of $\delta f$ is manifested as a factor of $e^{j 2 \pi \delta f t}$ in complex baseband. Over a symbol interval of length $T_{s}$, this offset causes a phase shift $e^{j 2 \pi \delta f T_{s}}=e^{j \delta \theta}$. In the framework of the general model (3), this means that

$$
B_{1}[n]=b_{1, n} e^{j n \delta \theta}
$$

Thus, the ratio

$$
A_{1}[n]=\frac{B_{1}[n]}{B_{1}[n-1]}=\epsilon^{j \delta \theta} \frac{b_{1, n}}{b_{1, n-1}}
$$

needed for DMMSE adaptation training the correlator c requires knowledge of $\delta \theta$ (which in turn requires knowledge of the frequency offset $\delta f$ ).

For an unknown $\delta \theta$, our strategy is to hypothesize several different values of $\delta \theta$, and to run DMMSE adaptations in parallel for each hypothesized value. The best value will then be chosen based on receiver statistics. In the context of the block algorithms described in Section 3.3, this corresponds to 
computing a different matrix $\hat{\mathbf{B}}$ for each possible hypothesized $\delta \theta$. However, computation of the correlation matrix $\hat{\mathbf{R}}$ and its inverse, which is often the most expensive step, is the same as that required for a situation in which the frequency offset is known. Moreover, this correlation matrix computation is the same for all users being received, while the $\hat{\mathbf{B}}$ computation is user-specific.

We use computer simulations to verify the performance of the preceding hypothesis based frequency acquisition scheme proposed in previous chapters. We consider, for simplicity of illustration, a synchronous CDMA system with five active users, each transmitting a binary phase-shift keying (BPSK) modulated signal. The processing gain (i.e., the number of chips per bit) is 10 , and short spreading sequences are employed. The value of the carrier frequency offset for each user is one-fifth of the data rate.

We consider a packetized system with a packet containing 500 bits: 50 training bits and 450 data bits. No decision-directed updates are performed in the simulations. Rather, training occurs at the beginning of each packet and values "learned" during training are used for demodulation throughout the duration of the packet.

Simulations of the (uncoded) BER are performed with a signal-to-noise ratio $\left(\frac{E b}{N_{0}}\right)$ for the desired user of $20 \mathrm{~dB}$. Since closed loop power control on a satellite link with a large round trip delay may be difficult, we evaluate our design under a severe nearfar problem, with two interferers transmitting at a power $20 \mathrm{~dB}$ stronger than the desired user, two interferers transmitting at the same power as the desired user. In Figure 7, we plot, for each value of $\frac{E_{b}}{N_{0}}$, the BER averaged over 1000 packets. It is clear from the figure that the performance of the receiver is insensitive to the near-far problem, and that the $10 \%$ uncoded BER required for good turbo code performance can be achieved at $\frac{E_{b}}{N_{0}}$ smaller than $5 \mathrm{~dB}$.

\section{Joint Source-Channel Coding}

In this paper, our focus is on source-channel coding for image data. Instead of considering the physical layer, as done in the previous two sections, we consider source-channel coding at the data transport layer, for end-to-end quality over a packetized net- work. Due to space restrictions, numerical results are omitted, and only a sketch of the basic ideas is provided. See [15] for details. Joint source-channel coding strategies for the physical layer of a satellite link is a subject of our ongoing research, and will be reported on in future publications.

Typical image/video bitstreams are highly structured i.e., characterized by a natural hierarchy of importance layers or resolutions. In fact, there is a strong push in the evolving next-generation image compression standard, JPEG-2000, to support bit stream scalability (bit stream scalability refers to the ability to be able to decode from the same stream to better and better qualities as more bits are decoded) in order to support a wide range of bit rates corresponding to available user bandwidths. The state-of-the-art image coder, 2D-SPIHT [16] produces a fully progressive code which means that if the transmission is stopped at any point, a lower bit image can still be decompressed and reconstructed. A longer prefix of the bit stream simply yields a higher reconstructed image quality when decoded. The reconstructed quality for such hierarchical bitstreams depends on which portions of the bitstream are reccived. In particular, if the first chunk of the stream is lost, then the remainder is essentially useless. There is thus the need for an efficient mechanism that converts a scalable, prioritized bitstream into a non-prioritized one so that is is better "matched" to the existing infrastructure.

Multiple description (MD) source encoding has recently emerged as an attractive framework for robust transmission over channels with transient shutdown characteristics. Examples of such channels are found in best-effort heterogeneous packet networks such as the Internet, where congestion and routing delays can lead to "holes" in the transmitted data stream corresponding to lost packets, or wireless systems during a deep fade. For example, In the current deployment of packet networks, switches in the network are typically oblivious to the structure or content of the packets that they process and switch. Packets that are more important to the application are treated identically to those that are not so important. In the following, we describe some recent work that illustrates some key ideas for applying source-channel coding on an end-to-end basis.

The basic idea in MD coding is to generate multiple independent descriptions of the source such that each description independently describes the 
source with a certain fidelity, and when more than one description is available, they can be synergistically combined to enhance the quality. The generalized ( $n$-channel) MD system can be informally characterized as a system that guarantees a hierarchy of delivered signal quality levels depending on how many descriptions $k \leqslant n$ are correctly received as opposed to a traditional multiresolution (MR) system for which the quality delivered depends which $k$ out of $n$ layers are received.

We consider a mechanism to transform a scalable source bit stream into a robust MD packet stream by encoding source "layers" of decreasing importance with progressively weaker forward error correction (FEC) [17] channel codes. Given the channel state and the transmission rate constraint, the question of how much protection to assign to each layer so as to maximize the expected quality at the receiver needs to be answered and we propose a nearly optimal solution to this problem. The major contribution of this approach is the rate-distortion "optimal" allocation between source and channel rates in the context of a best-effort packet network scenario. In the following, we describe the mechanics of the packetization strategy that converts the prioritized MR bitstream into an unprioritized MD stream using efficient erasure channel codes.

The quality profile reflects the target quality (or equivalently distortion $d$ : lower distortion implies higher quality and vice versa) when any $k$ out of $n$ descriptions are received. We will use the notation $\mathbf{d}(k))$ to describe the quality profile where the $i$ th entry in $\mathbf{d}(k)$ represents the target quality when $i$ descriptions are received.

Given $n$ and $\mathbf{d}(k)$, and a progressive bitstream, the stream is marked at $n$ different positions (see Figure 8) which correspond to the attainment of the distortion levels $\mathbf{d}(k)$, and is thus partitioned into $n$ sections or resolution layers. The $i$ th layer should be decodable when $i$ descriptions get through i.e., the number of erasures does not exceed $n-i$. This is attainable with the use of the family of ReedSolomon erasure-correction block codes ${ }^{1}$, which are characterized by the "optimal" code parameters $(n, i$, $n-i+1)$ which can correct any $(n-i)$ erasures out of $n$ descriptions. We split the $i$ th quality layer into

\footnotetext{
${ }^{1} \Lambda \mathrm{n}(n, k, d)$ block code is defined by a length $n$ code with $k$ user symbols and a minimum distance of $d$, i.e it can correct $(d-1)$ erasures. Reed Solomon block codes have the property of maximum distance $(d=n-k+1)$ i.e the whole data can be recovered from any $k$ out of $n$ symbols.
}

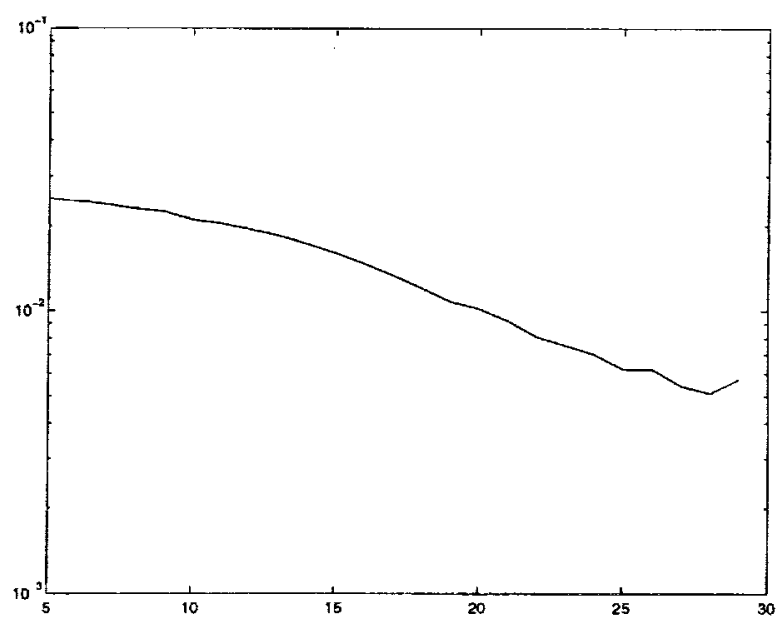

Figure 7: BER versus $E_{b} / N_{0}$ in a near-far setting

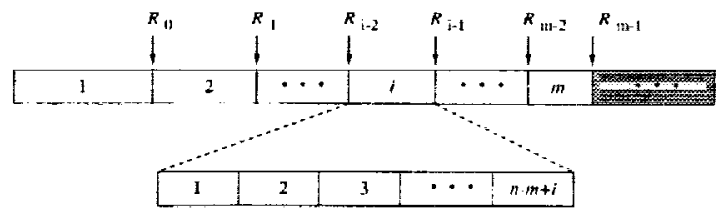

Figure 8: Progressive bitstream from the source coder partitioned into $m$ layers or quality levels.

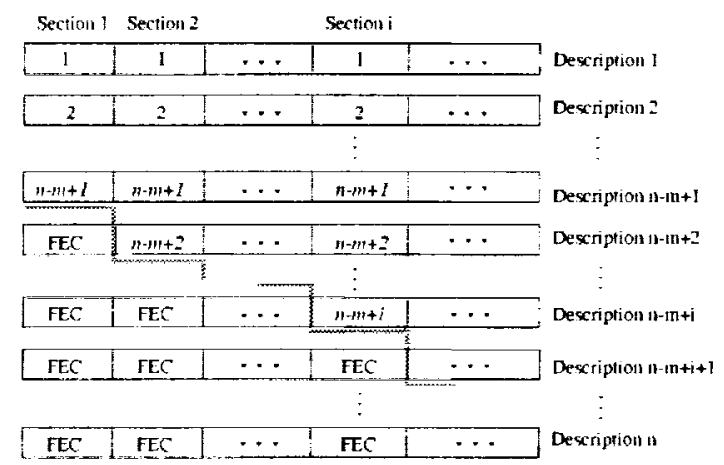

Figure 9: Forward Error Correction codes based MD codes for a quality profile of order $n$, i.e. having $n$ quality levels or "sections." 
$i$ equal parts, and apply the $(n, i, n-i+1)$ ReedSolomon code to get the "contribution" from the $i$ th level or section to each of the $n$ descriptions. The "contributions" from each of the $n$ quality levels or sections are concatenated to form the $n$ descriptions (see Figure 9). Thus, every description contains all $n$ layers, and all $n$ descriptions are "equal" in information content as intended.

\section{References}

[1] U. Madhow and M. L. Honig, "Mmse interference suppression for direct-sequence spreadspectrum cdma," IEEE Transactions on Communications, vol. 42 , no. 12 , pp. $3178-3188$, December 1994.

[2] U. Madhow, "Mmse interference suppression for timing acquisition and demodulation in direct-sequence cdma systems," IEEE Transactions on Communications, vol. 46 , no. $8, \mathrm{pp}$. 1065-1075, August 1998.

[3] C. Madhow, "Blind adaptive interference suppression for direct-sequence cdma," Proceedings of the IEEE, vol. 86, no. 10, pp. 2049 2069, October 1998.

[4] L. J. Zhu U. Madhow and L. Galup, "Differential mmse: new adaptive algorithms for equalization, interference suppression, and beamforming," Proceedings 32nd Asilomar Conf. Signals, Systems and Computers (Asilomar'98), October Pacific Grove, CA, 1998.

[5] Bixio Rimoldi and Quinn Li, "Coded continuous phase modulation using ring convolutional codes," IEEE Transactions on Communications, vol. 43 , no. 11 , pp. 2714-2720, Nov. 1995.

[6] Tom Richardson and Rüdiger Urbanke, "The capacity of low-density parity check codes under message-passing decoding," IEEE Trans. Inform. Theory, 1999, submitted.

[7] Giuseppe Caire, Giorgio Taricco, and Ezio Biglieri, "Bit-interleaved coded modulation," IEEE Trans. Inform. Theory, vol. 44, no. 3, pp. 927-946, May 1998.
[8] Bixio E. Rimoldi, "A decomposition approach to CPM," IEEE Trans. Inform. Theory, vol. 34 , no. 2, pp. 260-270, March 1988.

[9] L. R. Bahl, J. Cocke, F. Jelinek, and J. Raviv, "Optimal decoding of linear codes for minimizing symbol error rate," IEEE Trans. Inform. Theory, vol. IT-20, pp. 284-287, Mar. 1974.

[10] J. B. Anderson, T. Aulin, and C.-E. Sundberg, Digital Phase Modulation, Applications of communications theory. Plenum Press, New York, 1986.

[11] S. Haykin, Adaptive Filter Theory, Prentice Hall, 1991.

[12] Golub and Van Loan, Matrix Computations, Johns Hopkins University Press, Baltimore, MD, 1996.

[13] U. Madhow, "Blind adaptive interference suppression for the near-far resistant acquisition and demodulation of direct-sequence cdma signals," IEEE Transactions on Signal Processing, vol. 45, no. 1, pp. 124-136, January 1997.

[14] D. Warrier and U. Madhow, "Noncoherent communication in space and time," Proc. 1999 Conference on Information Sciences and Systems (CISS'99), March Baltimore, MD, 1999, (journal paper submitted to the IEEE Trans. on Information Theory, and available at http://www.comm.csl.uiuc.edu/ madhow).

[15] Kang-Won Lee, R. Puri, K. Ramchandran, and V. Bharghavan, "An integrated source coding and congestion control framework for video streaming in the internet," To appear in IEEE INFOCOM, March, Tel-Aviv, 2000.

[16] A. Said and A. Pearlman, W, "A new fast and efficient image codec based on set partitioning in hierarchical trees," IEEE Transactions on Circuit Systems and Video Technology, vol. 6, pp. 243-250, June 1996.

[17] F. J. Macwilliams and N. J. A. Sloane, The Theory of Error Correcting Codes, ElseiverNorth-Holland, 1977. 

Davis Highway, Suite 1204, Arlington, VA 22202-4302, and to the Office of Management and Budget. Paperwork Reduction Project (0704-0188), Washington, DC 20503.

\begin{tabular}{|l|c|c|c|}
\hline 1. AGENCY USE ONLY (Leave blank) & $\begin{array}{c}\text { 2. REPOAT DATE } \\
\text { June } 2000\end{array}$ & $\begin{array}{r}\text { 3. REPORT TYPE AND DATES COVERED } \\
\text { Technical Memorandum }\end{array}$ \\
\hline 4. TITLE AND SUBTITLE & 5. FUNDING NUMBERS
\end{tabular}

4. TITLE AND SUBTITLE

A Wideband Satcom Based Avionics Network With CDMA Uplink and TDM Downlink

6. AUTHOR(S)

D. Agrawal, B.S. Johnson, U. Madhow, K. Ramchandran, and K.S. Chun
WU $-576-01-21-00$

8. PERFORMING ORGANIZATION REPORT NUMBER

E-12259

National Aeronautics and Space Administration

John H. Glenn Research Center at Lewis Field

Cleveland, Ohio 44135-3191

9. SPONSORING/MONITORING AGENCY NAME(S) AND ADDRESS(ES)

10. SPONSORING/MONITORING AGENCY REPORT NUMBER

National Aeronautics and Space Administration

Washington, DC 20546-0001

NASA TM-2000-210057

11. SUPPLEMENTARY NOTES

Prepared for the 18th International Communications Satellite Systems Conference and Exhibit sponsored by the American Institute of Aeronautics and Astronautics, Oakland, California. April 10-14, 2000. D. Agrawal and B.S. Johnson, ECE Department and Coordinated Science Laboratory, University of Illinois, Urbana Champaign, Urbana, Ilinois 61801; U. Madhow, ECE Department, University of California, Santa Barbara, 552 University Avenue, Santa Barbara, Califomia 93106-0002; K. Ramchandran, EECS Department, University of California, Berkeley, Berkeley, Califormia 94720; Work funded under NASA Grant NAG3-2263. K.S. Chun, NASA Glenn Research Center. Responsible person, K.S. Chun, organization code 5650, (216) 433-3624.

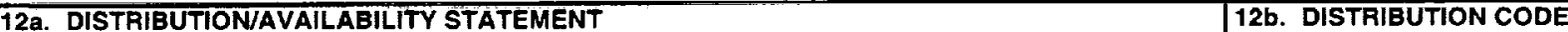

Unclassified - Unlimited

Subject Category: 32

Distribution: Nonstandard

This publication is available from the NASA Center for AeroSpace Information, (301) 621-0390.

13. ABSTRACT (Maximum 200 words)

The purpose of this paper is to describe some key technical ideas behind our vision of a future satcom based digital communication network for avionics applications The key features of our design are as follows: (a) Packetized transmission to permit efficient use of system resources for multimedia traffic; (b) A time division multiplexed (TDM) satellite downlink whose physical layer is designed to operate the satellite link at maximum power efficiency. We show how powerful turbo codes (invented originally for linear modulation) can be used with nonlinear constant envelope modulation, thus permitting the satellite amplifier to operate in a power efficient nonlinear regime. (c) A code division multiple access (CDMA) satellite uplink, which permits efficient access to the satellite from multiple asynchronous users. Closed loop power control is difficult for bursty packetized traffic, especially given the large round trip delay to the satellite. We show how adaptive interference suppression techniques can be used to deal with the ensuing near-far problem. (d) Joint source-channel coding techniques are required both at the physical and the data transport layer to optimize the end-to-end performance. We describe a novel approach to multiple description image encoding at the data transport layer in this paper.

14. SUBJECT TERMS

ATN; Satcom; Avionics, Network CDMA

15. NUMBER OF PAGES 17

16. PRICE CODE

$\mathrm{A} 03$

\begin{tabular}{|c|c|c|}
\hline $\begin{array}{c}\text { 17. SECURITY CLASSIFICATION } \\
\text { OF REPORT } \\
\text { Unclassified }\end{array}$ & $\begin{array}{c}\text { 18. SECURITY CLASSIFICATION } \\
\text { OF THIS PAGE } \\
\text { Unclassified }\end{array}$ & $\begin{array}{c}\text { 19. SECURITY CLASSIFICATION } \\
\text { OF ABSTRACT } \\
\text { Unclassified }\end{array}$ \\
\hline
\end{tabular}

NSN 7540-01-280-5500

\section{LIMITATION OF ABSTRACT}

Standard Form 298 (Rev. 2-89) Prescribed by ANSI Std. Z39-18 298-102 